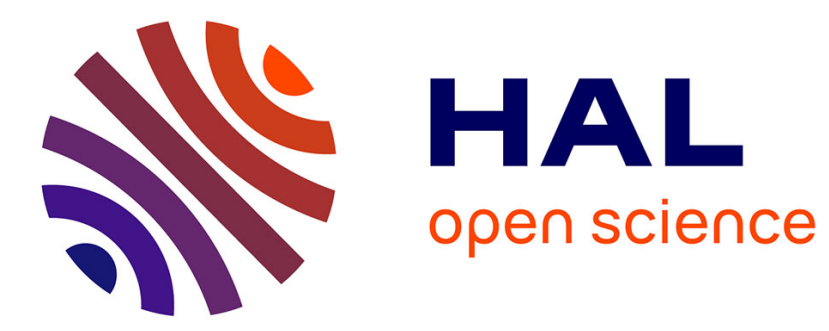

\title{
An approximate controllability result with continuous spectrum: the Morse potential with dipolar interaction
} Nabile Boussaid, Marco Caponigro, Thomas Chambrion

\section{To cite this version:}

Nabile Boussaid, Marco Caponigro, Thomas Chambrion. An approximate controllability result with continuous spectrum : the Morse potential with dipolar interaction. SIAM Conference on Control and its applications, Jul 2015, Paris, France. hal-01143308

\section{HAL Id: hal-01143308 \\ https://hal.science/hal-01143308}

Submitted on 17 Apr 2015

HAL is a multi-disciplinary open access archive for the deposit and dissemination of scientific research documents, whether they are published or not. The documents may come from teaching and research institutions in France or abroad, or from public or private research centers.
L'archive ouverte pluridisciplinaire HAL, est destinée au dépôt et à la diffusion de documents scientifiques de niveau recherche, publiés ou non, émanant des établissements d'enseignement et de recherche français ou étrangers, des laboratoires publics ou privés. 


\title{
An approximate controllability result with continuous spectrum : the Morse potential with dipolar interaction*
}

\author{
Nabile Boussaï ${ }^{\dagger} \quad$ Marco Caponigro ${ }^{\ddagger} \quad$ Thomas Chambrion $^{\S}$
}

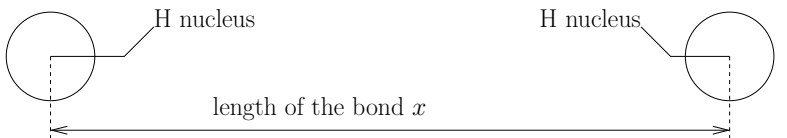

Figure 1: Schematic representation of a $\mathrm{H}-\mathrm{H}$ bond

\begin{abstract}
This note presents a positive approximate controllability result for a bilinear quantum system modeling a chemical bond. The main difficulties are due to the presence of a continuous spectrum part for the uncontrolled Hamiltonian (modeled by a Morse potential) and the unboundedness of the interaction potential (dipolar interaction). The proof is based on averaging technique and spectral analysis.
\end{abstract}

\section{Problem Specification.}

1.1 Physical motivation In this note, we consider the dynamics of a molecular bond, such as a $\mathrm{H}-\mathrm{H}$ bond in a dihydrogen molecule (Figure 1), modeled by a bilinear Schrödinger equation

$$
\mathrm{i} \frac{\hbar}{2 m} \frac{\partial \psi}{\partial t}=[(-\Delta+V(x))+u(t) W(x)] \psi(x, t)
$$

where $\hbar=\frac{h}{2 \pi}$ is the reduced Planck constant, $m$ is the reduced mass of the system, $x>0$ is the length of the bond between the atomic nuclei, $\Delta$ is the Laplacian (with Dirichlet boundary conditions) on $(0,+\infty), V$ : $(0,+\infty) \rightarrow \mathbf{R}$ is the potential of the free dynamics (without external interaction) and $W:(0,+\infty) \rightarrow \mathbf{R}$ is the interaction potential, modeling the influence of an electric field (e.g., a laser) on the molecule. The function $u:[0,+\infty) \rightarrow \mathbf{R}$ is the intensity of the external field. The wave function $\psi \in L^{2}((0,+\infty), \mathbf{C})$ determines

\footnotetext{
*Supported by European Research Council ERC StG 2009 "GeCoMethods", contract number 239748.

†Laboratoire de Mathématiques de Besancon 16, route de Gray - 25030 Besancon Cedex, France

$\ddagger$ Équipe M2N - EA 7340, CNAM, 292 Rue Saint Martin, 75003, Paris, France.

$\S$ Université de Lorraine, IECL, 54619 Vandœuvre-lès-Nancy, and CNRS, IECL, 54619 Vandœuvre-lès-Nancy and Inria, 54619 Vandœuvre-lès-Nancy, France.
}

the quantum state of the system. For instance, the probability for the molecular bond to have length in $\left[l_{0}, l_{1}\right]$ at time $t$ is $\int_{l_{0}}^{l_{1}}|\psi(x, t)|^{2} \mathrm{~d} x$.

In this note, we focus on the case where $V$ : $x \mapsto D_{e}\left(1-e^{-a\left(x-x_{e}\right)}\right)^{2}$ is the celebrated Morse potential [18]. With an abuse of notation we identify the function $V$ with the multiplicative operator $\psi \mapsto V \psi$ in $L^{2}((0,+\infty), \mathbf{C})$. The constants $D_{e}$ and $a$ represent, respectively, the depth and the width of the potential well, the length $x_{e}$ is the equilibrium length of the bond. For a dihydrogen molecule in standard conditions, $D_{e} \approx 38292 \mathrm{~cm}^{-1}, a \approx 1.4426$ and $x_{e} \approx 7.741410^{-11} \mathrm{~m}$ (see [13]). The Morse potential is nowadays essentially used for academic purposes and it has been replaced in practical applications by more realistic potentials, see for instance, [13, page 65] and [15]. It has the advantage of a complete explicit analytic description of the eigenstates of the free Hamiltonian. Indeed, the operator $-\Delta+V$ admits a finite number of simple eigenvalues

$$
\lambda_{j}=h \nu_{0}\left(j+\frac{1}{2}\right)-\frac{\left[h \nu_{0}\left(j+\frac{1}{2}\right)\right]^{2}}{4 D_{e}}
$$

where $\nu_{0}=\frac{a}{2 \pi} \sqrt{2 D_{e} / m}$ and $j=1, \ldots,\left\lfloor 2 D_{e} /\left(h \nu_{0}\right)\right\rfloor-1$. The associated eigenfunctions $\phi_{j}$ may be expressed in terms of Laguerre polynomials. A direct computations shows that, for every $j \neq k$,

$$
\left\langle\phi_{j}, x \phi_{k}\right\rangle \neq 0 \text {. }
$$

In the present analysis we consider as control operator $W(x)=x$. We consider controls $u:[0, T] \rightarrow$ $[0,+\infty)$ which are piecewise constant. To define the solution of (1.1) associated with an admissible control we consider, for every constant $u \geq 0$, the operator $-\Delta+V+u W$ which is the generator of a unitary group on $L^{2}((0,+\infty), \mathbf{C})$, hence one can define the solution of (1.1) for every initial condition. By concatenation, one defines the solution $t \mapsto \Upsilon_{t, 0}^{u} \psi_{0}$ of (1.1) for every initial condition $\psi_{0}$, for every piecewise constant function $u$ with value in $[0,+\infty)$.

1.2 Control problem An important question for applications is the existence and the efficient design of 
controls $u$ steering the wave function $\psi$ from a given initial state $\psi_{0}$ to a given desirable target $\psi_{f}$, with a particular focus on the transfer from one energy level to another.

This question has been tackled by many authors, using different approaches, in the last decades. The linear test $[2,3,4]$, Lyapunov approach $[17,5,20,19,6]$ were used to give a precise description of the attainable set of toy models of (1.1) where $x$ belongs to a compact set (and $-\Delta+V$ thus has a discrete spectrum). Very few works have considered the actual form of (1.1), including the continuous part of the spectrum of $-\Delta+$ $V$. Let us cite [16] (Lyapunov techniques) and [9] (averaging techniques). In both cases, the function $W$, and hence the associated multiplication operator in $L^{2}((0,+\infty), \mathbf{C})$, was assumed to be bounded.

Results of approximate simultaneous controllability in a more general framework (in the sense of Section 2 below) using piecewise constant controls have been firstly introduced in [10] and then refined following works (see, for instance, [7] and [8]).

1.3 Contribution Combining averaging techniques and spectral theory, we prove the simultaneous approximate controllability of (1.1) in the space spanned by the two first eigenvectors. More precisely we have the following result.

Proposition 1.1. Let $W: x \mapsto x$. There exists a sequence of piecewise constant functions $u_{n}$ : $\left[0, T_{n}\right] \rightarrow[0,+\infty)$ such that the two sequences $\left(\left|\left\langle\phi_{2}, \Upsilon_{T_{n}, 0}^{u_{n}} \phi_{1}\right\rangle\right|\right)_{n \in \mathbf{N}}$ and $\left(\left|\left\langle\phi_{1}, \Upsilon_{T_{n}, 0}^{u_{n}} \phi_{2}\right\rangle\right|\right)_{n \in \mathbf{N}}$ tend to one as $n$ tends to $+\infty$.

To the best of our knowledge, this result is the first positive approximate controllability result of a bilinear quantum dynamics when the interaction potential $W$ is unbounded and the spectrum of the free Hamiltonian $-\Delta+V$ has a continuous part.

1.4 Content In Section 2, we introduce our assumptions in an abstract framework that will be used as the "black box" requirements in our application. The idea is that the interested reader can include a variety of other examples based on this template. Thus this framework includes the dynamics (1.1) with $W: x \mapsto x$. In Section 3 , we recall some averaging results for bilinear quantum systems when there exists a Hilbert basis made of eigenvectors of the free Hamiltonian. In Section 4, we first use spectral analysis in Section 4.1 to study the discrete spectrum of approximations of the dynamics (1.1). Then, we use the results of Section 3 in Section 4.2 to prove Proposition 1.1.

\section{Abstract framework}

2.1 Assumption and notations In the separable Hilbert space $H$, we consider the abstract evolution equation

$$
\frac{d \psi}{d t}=(A+u(t) B) \psi
$$

where the operators $A$ and $B$ satisfies the following

Assumption 1. 1. the operator $A$ is skew-adjoint with domain $D(A)$;

2. the spectrum of $A$ splits in a discrete part $\left\{-\mathrm{i} \lambda_{1},-\mathrm{i} \lambda_{2}, \ldots\right\}$ and a (possibly essential) part included in $-\mathrm{i}[l,+\infty)$, where $l>0$ and $\left(\lambda_{j}\right)$ is a (finite or infinite) increasing sequence of $(0, l)$;

3. the operator $B$ is skew-adjoint with i $B$ nonnegative;

4. the transition $(1,2)$ is weakly non-degenerate: that is, the eigenvectors $\phi_{1}^{0}$ and $\phi_{2}^{0}$ of $A$ associated with eigenvalues $-\mathrm{i} \lambda_{1}$ and $-\mathrm{i} \lambda_{2}$ belong to the domain of $B,\left\langle\phi_{1}^{0}, B \phi_{2}^{0}\right\rangle \neq 0$ and $2 \lambda_{2}-\lambda_{1} \notin \cup_{j \geq 3}\left\{\lambda_{j}\right\} \cup$ $[l,+\infty)$;

5. for every $u>0, A+u B$ has compact resolvent (and thus discrete spectrum) and every eigenvector of $A+u B$ belongs to the intersection of the domains of $A$ and $B$.

From these assumptions we deduce that for every $u \geq 0, \mathrm{i}(A+u B)$ is bounded from below. Moreover for every $u>0$, the form domain $Q(\mathrm{i} A+u \mathrm{i} B)$ of $\mathrm{i} A+u \mathrm{i} B$ is included in the form domain $Q(\mathrm{i} u B)$ of $\mathrm{i} B$ and $\mathrm{i} A+u \mathrm{i} B \geq \mathrm{i} u B$ in the form sense.

For every $u>0, A+u B$ is a well-defined skewadjoint operator and its form domain is $Q(A) \cap Q(B)$ : the intersections of the form domains of $A$ and $B$, see [21, Theorem VIII.15], as the associated quadratic form is a closed positive quadratic form. Since the form domain is invariant by the unitary group $e^{t(A+u B)}$, by interpolation see [1, Section 2.8], the space $Q(A) \cap Q(B)$ is invariant by $e^{t(A+u B)}$ for any $u>0$.

We denote by $\phi_{1}^{0}, \phi_{2}^{0}, \ldots, \phi_{n}^{0}$ an orthonormal family of eigenvectors of $A$ associated with the family of eigenvalues $\left\{\mathrm{i} \lambda_{1}, \mathrm{i} \lambda_{2}, \ldots, \mathrm{i} \lambda_{n}\right\}$, and with $\left(\phi_{j}^{u}\right)_{j \in \mathbf{N}}$ a Hilbert basis of $H$ made of eigenvectors of $A+u B$ associated with the family of eigenvalues $\left(\left(-\mathrm{i} \lambda_{j}^{u}\right)\right)_{j \in \mathbf{N}^{*}}$.

Proposition 2.1. The dynamics (1.1) fits within the frame of Assumption 1 with

$$
\begin{aligned}
& \text { 1. } A=\mathrm{i}(-\Delta+V) \text { with domain } H^{2}(0,+\infty) \cap \\
& \left.H_{0}^{1}(0,+\infty) \text { (Dirichlet boundary conditions at } 0\right) \text {; }
\end{aligned}
$$


2. $B=-\mathrm{i} W$, where $W(x)=x$, with its maximal domain $\left\{\psi \in L^{2}(0,+\infty), B \psi \in L^{2}(0,+\infty)\right\}$;

3. the constants

$$
-\frac{\sqrt{16 D_{e}^{2}-56 h \nu_{0} D_{e}+41 h^{2} \nu_{0}^{2}}-4 D_{e}+h \nu_{0}}{2 h \nu_{0}}
$$

and

$$
\frac{\sqrt{16 D_{e}^{2}-56 h \nu_{0} D_{e}+41 h^{2} \nu_{0}^{2}}+4 D_{e}-h \nu_{0}}{2 h \nu_{0}}
$$

are not integers larger than 3 and smaller than $\left\lfloor 2 D_{e} /\left(h \nu_{0}\right)\right\rfloor$, and

$$
56 h \nu_{0} D_{e}-41 h^{2} \nu_{0}^{2}<16 D_{e}^{2} .
$$

Proof. Assumptions 1.1 and 1.2 are immediate as $V-D_{e}$ is a Kato-Rellich perturbation of $-\Delta+D_{e}$. Assumptions 1.3 is straightforward.

To check the first part of Assumption 1.4, one computes $\left\langle\phi_{1}, B \phi_{2}\right\rangle$, which is not zero (see [11, Eq. (21)]).

To check the second part of Assumption 1.4, one computes

$$
2 \lambda_{2}-\lambda_{1}=\frac{56 h \nu_{0} D_{e}-41 h^{2} \nu_{0}^{2}}{16 D_{e}}
$$

and one solves $2 \lambda_{2}-\lambda_{1}=\lambda_{j}$ for $j \in \mathbf{N}$. The solutions (if any) are

$$
j_{1}=-\frac{\sqrt{16 D_{e}^{2}-56 h \nu_{0} D_{e}+41 h^{2} \nu_{0}^{2}}-4 D_{e}+h \nu_{0}}{2 h \nu_{0}}
$$

and

$$
j_{2}=\frac{\sqrt{16 D_{e}^{2}-56 h \nu_{0} D_{e}+41 h^{2} \nu_{0}^{2}}+4 D_{e}-h \nu_{0}}{2 h \nu_{0}} .
$$

The non-degeneracy hypothesis is verified as soon as both $j_{1}$ and $j_{2}$ are not integers larger than 3 and smaller than $\left\lfloor 2 D_{e} /\left(h \nu_{0}\right)\right\rfloor$, and $2 \lambda_{2}-\lambda_{1}<0$. These conditions are verified for the $\mathrm{H}-\mathrm{H}$ molecule $\left(\lambda_{1}=\right.$ $D_{e}-103.2 \mathrm{kcal} / \mathrm{mol}, \lambda_{2}=D_{e}-91.4 \mathrm{kcal} / \mathrm{mol}$, hence $2 \lambda_{2}-\lambda_{1}=D_{e}-79.6 \mathrm{kcal} / \mathrm{mol}$ thus $2 \lambda_{2}-\lambda_{1}<D_{e}$ and $\lambda_{3}=D_{e}-80.3 \mathrm{kcal} / \mathrm{mol}<2 \lambda_{2}-\lambda_{1}<\lambda_{4}=D_{e}-69.8$ $\mathrm{kcal} / \mathrm{mol})$.

The proof of Assumption 5 follows from [22, Theorem XIII.64.(iv)]. Indeed the unit ball associated to the form $-\Delta+V+u W$ is included in $H_{0}^{1}(0, \infty)$ and in the unit ball form the norm $u \mapsto\|\sqrt{x} u\|$ which provides the localization needed for the Rellich-Kondrachov theorem. The fact that the eigenvectors are in the domain of $B$ comes from the exponential decay of eigenvectors [12, Theorem 4.1]. Then since $H^{2}(0,+\infty)$ is the maximal domain of the Laplacian it follows that the eigenvectors are in $H^{2}(0,+\infty)$. The boundary condition is contained in the form domain definition.
Let $\Phi=\left(\phi_{j}\right)_{j \in \mathbf{N}}$ be a Hilbert basis of $H$ contained in the domains of $A$ and $B$ (such a basis exists by Assumption 1.5). For every $N$ in $\mathbf{N}$, denote by $\mathcal{L}_{N}^{\Phi}$ the linear space spanned by $\phi_{1}, \phi_{2}, \ldots, \phi_{N}$ and by $\pi_{N}^{\Phi}: H \rightarrow$ $\mathcal{L}_{N}^{\Phi}$ the associated orthogonal projection. We define the compression of $A$ (resp. $B$ ) in basis $\Phi$ at order $N$ as the linear operator $A_{N}^{\phi}=\pi_{N}^{\Phi} A_{\left\lceil\mathcal{L}_{N}^{\Phi}\right.}\left(\operatorname{resp} . B_{N}^{\phi}=\pi_{N}^{\Phi} B_{\left\lceil\mathcal{L}_{N}^{\Phi}\right.}\right)$.

2.2 Definition of propagators In general, Assumption 1 is not enough to define the solutions of (2.3) for usual functional classes such as continuous or locally integrable functions $u$. We restrict ourselves to the much smaller class of piecewise constant functions:

Definition 1. A function $u:[0,+\infty) \rightarrow \mathbf{R}$ is piecewise constant if there exists an increasing unbounded sequence $\left(t_{i}\right)_{i \in \mathbf{N}}$ such that $u_{\mid\left(t_{i}, t_{i+1}\right)}$ is constant (with value denoted $u_{i}$ ) for every $i \in \mathbf{N}$.

Let $(A, B)$ satisfy Assumption 1. For every piecewise constant function $u:[0,+\infty) \rightarrow[0,+\infty)$, we define the propagator $t \in[0,+\infty) \mapsto \Upsilon_{t}^{u}$ of $(2.3)$ by concatenation

$$
\Upsilon_{t}^{u}=e^{\left(t-t_{l}\right)\left(A+u_{l} B\right)} \circ \cdots \circ e^{t_{1}\left(A+u_{0} B\right)}
$$

for $t$ belonging to $\left[t_{l}, t_{l+1}\right)$. For every $s, t \geq 0$, we define also

$$
\Upsilon_{t, s}^{u}=\Upsilon_{t}^{u} \circ\left(\Upsilon_{s}^{u}\right)^{-1}
$$

Notice that if $u>0$ then $\Upsilon_{t}^{u}$ leaves invariant $Q(A) \cap Q(B)$.

Using Assumption 1.5, we can prove the strong continuity of the map $u \mapsto \Upsilon_{t}^{u}$ for any fixed $t$ : For any $\psi$ and $\phi$ in $Q(A) \cap Q(B)$ we have for any positive piecewise constant functions $u$ and $v$

$\left\langle\psi, \Upsilon_{t}^{u} \phi\right\rangle-\left\langle\psi, \Upsilon_{t}^{v} \phi\right\rangle=\int_{0}^{t}\left\langle\psi, \Upsilon_{s}^{u}(u(s)-v(s)) B \Upsilon_{s}^{v} \phi\right\rangle d s$

which provides the continuity of $u \mapsto\left\langle\psi, \Upsilon_{t}^{u} \phi\right\rangle$ in the $L^{1}$ norm whenever we consider positive piecewise constant functions. For $\phi$ and $\psi$ in $H$ we conclude by density and thus obtain the weak continuity. The general case of non-negative piecewise constant functions follows from the strong convergence of $e^{t(A+\delta B)}$ to $e^{t A}$ as $\delta \rightarrow 0$ as $A+\delta B$ tends to $A$ in the strong resolvent sense. For this we have to use a symmetrized version of the resolvent expansion, see [14]. Finally, the strong continuity is obtained by the fact that the operators are unitary.

\section{Averaging techniques in quantum control}

3.1 Result The aim of this Section is to prove the following 
Proposition 3.1. Let $(A, B)$ satisfy Assumptions 1.1, 1.2, and 1.4. Assume that there exists a Hilbert basis $\left(\phi_{j}\right)_{j \in \mathbf{N}}$ of $H$ made of eigenvectors of $A$ contained in the domain of $B$. Let $T=2 \pi /\left|\lambda_{2}-\lambda_{1}\right|$ and $u_{n}$ be $a$ sequence of positive $T$-periodic piecewise constant functions converging in $L^{1}([0, T])$ to $u_{\infty}: t \mapsto 1+\sin \left(\left(\lambda_{2}-\right.\right.$ $\left.\left.\lambda_{1}\right) t\right)$. Then there exists $T^{*}$ such that the two sequences $\left(\left|\left\langle\phi_{2}, \Upsilon_{n T^{*}, 0}^{u_{n} / n} \phi_{1}\right\rangle\right|\right)_{n \in \mathbf{N}}$ and $\left(\left|\left\langle\phi_{1}, \Upsilon_{n T^{*}, 0}^{u_{n} / n} \phi_{2}\right\rangle\right|\right)_{n \in \mathbf{N}}$ tend to one as $n$ tends to infinity.

Notice that under the assumptions of Proposition 3.1, $A$ has no essential spectrum.

3.2 General principle For finite dimensional systems, averaging methods consist in replacing oscillating dynamics $\dot{y}=f(t, y)$ by its average $\dot{z}=\bar{f} z$ where $\bar{f}(z):=\lim \frac{1}{T} \int_{0}^{T} f(t, z) \mathrm{d} t$. When the dynamics $f$ is sufficiently regular, the solutions $y_{\epsilon}$ and $z_{\epsilon}$ of $\dot{y}=\epsilon f(t, y)$ and $\dot{z}=\epsilon \bar{f}(z)$ have similar behaviors (difference of the order of $\epsilon$ ) for time less than $1 / \epsilon$. Averaging theory has grown to a whole theory in itself, we refer to [23] for an introduction. In quantum mechanics, averaging theory has been extensively used (under the name of "Rotating Wave Approximation") since the 60's, for finite dimensional systems. Let us briefly recall the core ideas of the method.

Up to the change of variable $y=e^{-t A} x$, the system $\dot{x}=A+u(t) B x$ may be rewritten as $\dot{y}=u(t) e^{-t A} B e^{t A} y$. If $A$ is diagonal with diagonal entries $\left(i \lambda_{j}\right)_{1<j<N}$ and $B$ has entries $\left(b_{j k}\right)_{1<j, k \leq N}$, then $u(t) e^{-t A} B e^{t \bar{A}}$ has entries $\left(b_{j k} u(t) e^{\left(\lambda_{j}-\lambda_{k}\right) t}\right)_{1 \leq j, k \leq N}$. Choosing $u$ small enough and $T=2 \pi /\left(\lambda_{2}-\lambda_{1}\right)$ periodic such that

$$
\int_{0}^{T} b_{j k} u(t) e^{\left(\lambda_{j}-\lambda_{k}\right) t} \mathrm{~d} t=0, \quad 2 \leq j, k \leq N
$$

then the averaged system behaves like a rotation in the plane spanned by the first two eigenvectors of $A$. The non-degeneracy condition 1.4 is indeed a sufficient condition to ensure the existence of a periodic control $u$ satisfying the moment vanishing hypothesis (3.4).

\subsection{A simple yet wrong proof of Proposition 3.1} Extending the finite dimensional procedure described in Section 3.2 to the infinite dimensional setting of Proposition 3.1 is not completely straightforward. Our proof is inspired by [9, Theorem 1]. Let us assume for a while that (2.3) admits strong solutions for control $u$ of class $C^{1}$, that the propagator $u \mapsto \Upsilon^{u}$ is continuous with respect to the $L^{1}$ norm and that some Duhamellike formula is available. Projecting (2.3) on the $N$ - dimensional space $\mathcal{L}_{N}^{\Phi}$, we get

$$
\begin{array}{r}
\pi_{N}^{\Phi} \frac{d}{d t} \psi=\overbrace{\pi_{N}^{\Phi} A \pi_{N}^{\Phi}}^{A_{N}^{\Phi}} \psi+u(t) \overbrace{\pi_{N}^{\Phi} B \pi_{N}^{\Phi}}^{B_{N}^{\Phi}} \psi \\
+u(t) \pi_{N}^{\Phi} B\left(1-\pi_{N}^{\Phi}\right) \psi .
\end{array}
$$

Let $X^{u,(N)}$ be the propagator of the finite dimensional dynamics

$$
x^{\prime}=A_{N}^{\Phi} x+u(t) B_{N}^{\Phi} x .
$$

Using Duhamel formula, we deduce relation between the infinite dimensional propagator $\Upsilon^{u}$ and $X^{u,(N)}$ which is

$\pi_{N}^{\Phi} \Upsilon_{t}^{u} \psi_{0}=X_{t, 0}^{u,(N)} \psi_{0}+\int_{0}^{t} u(s) X_{t, s}^{u,(N)} B\left(1-\pi_{N}^{\Phi}\right) \Upsilon_{s}^{u} \psi_{0} \mathrm{~d} s$

Projecting again on $\mathcal{L}_{2}$ and recalling that $\pi_{2}^{\Phi}$ and $\pi_{N}^{\Phi}$ do commute we have

$$
\begin{aligned}
\pi_{2}^{\Phi} \Upsilon_{t}^{u} \psi_{0}= & \pi_{2}^{\Phi} X_{t, 0}^{u,(N)} \psi_{0} \\
& +\int_{0}^{t} u(s) \pi_{2}^{\Phi} X_{t, s}^{u,(N)} B\left(1-\pi_{N}^{\Phi}\right) \Upsilon_{s}^{u} \psi_{0} \mathrm{~d} s \\
= & \pi_{2}^{\Phi} X_{t, 0}^{u,(N)} \psi_{0} \\
& +\int_{0}^{t} u(s) X_{t, s}^{u,(N)} \pi_{2}^{\Phi} B\left(1-\pi_{N}^{\Phi}\right) \Upsilon_{s}^{u} \psi_{0} \mathrm{~d} s \\
& +\int_{0}^{t} u(s)\left[X_{t, s}^{u,(N)}, \pi_{2}^{\Phi}\right] B\left(1-\pi_{N}^{\Phi}\right) \Upsilon_{s}^{u} \psi_{0} \mathrm{~d} s .
\end{aligned}
$$

We choose now to use a control $u=u^{*} / n$ with $n$ large enough, where $u^{*}$ is a continuous function satisfying the vanishing moments condition (3.4). Then, by [9], there exists $T^{*}$ such that $X_{n T^{*}, 0}^{u,(N)}$ exchange $\phi_{1}$ and $\phi_{2}$, up to an arbitrary small error.

Notice, and this is crucial for our purpose, that the $L^{1}$ norm of $u$ on $\left[0, n T^{*}\right]$ is uniformly bounded in $n$ (since it converges to the $L^{1}$ norm of $u^{*}$ on $\left[0, T^{*}\right]$ ). As a consequence, the last two terms of (3.6) tend to zero as $N$ and $n$ tend to infinity. Indeed

$$
\begin{array}{r}
\left\|\int_{0}^{t} u(s) X_{t, s}^{u,(N)} \pi_{2}^{\Phi} B\left(1-\pi_{N}^{\Phi}\right) \Upsilon_{s}^{u} \psi_{0} \mathrm{~d} s\right\| \\
\leq\left\|\pi_{2}^{\Phi} B\left(1-\pi_{N}^{\Phi}\right)\right\| \int_{0}^{n T^{*}}|u(s)| \mathrm{d} s
\end{array}
$$

tends to zero as $N$ tends to infinity (uniformly in $n$ ) since $\left\|\pi_{2}^{\Phi} B\left(1-\pi_{N}^{\Phi}\right)\right\|=\left\|\left(1-\pi_{N}^{\Phi}\right) B \pi_{2}^{\Phi}\right\|$ tends to zero. While

$$
\left\|\int_{0}^{t} u(s)\left[X_{t, s}^{u,(N)}, \pi_{2}^{\Phi}\right] B\left(1-\pi_{N}^{\Phi}\right) \Upsilon_{s}^{u} \psi_{0} \mathrm{~d} s\right\|
$$


tends to zero as $n$ tends to infinity (for any fixed $N$ ) by finite dimensional averaging.

In conclusion, $\pi_{2}^{\Phi} \Upsilon_{n T^{*}}^{u} \psi_{0}$ tends to $\pi_{2}^{\Phi} X_{n T^{*}, 0}^{u^{*} / n,(N)} \psi_{0}$ as $n$ tends to infinity which is the expected result if $u_{n}=u^{*}$ for every $n$. If $u_{n} \neq u^{*}$ but $\left(u_{n}\right)_{n}$ tends to $u^{*}$ in $L^{1}$ sense, the conclusion follows from the continuity of the propagators.

3.4 Two major flaws The sketch of proof presented in Section 3.3 is not correct for the following reasons.

The first reason is that the only way to guarantee the vanishing moments condition (3.4) for any $N$ is to use $u^{*}$ in a sine form, for instance $u^{*}(t)=1+$ $\sin \left(\left(\lambda_{2}-\lambda_{1}\right) t\right)$, however in the framework of Assumption 1 the propagators are defined only for piecewise constant functions (Section 2.2).

The second flaw in the proof of Section 3.3 is that in general, even for piecewise constant control function, (2.3) does not admit strong solutions, only a very weak notion of solutions (where the test vector are taken in the space spanned by the eigenvectors of $A$ ). In particular, there is no hope, in general, to prove a Duhamel-like formula for this dynamics.

3.5 Proof of Proposition 3.1 For the ease of notation, we introduce $T^{*}=\frac{\pi}{\left\langle\left\langle\phi_{1}, B \phi_{2}\right\rangle\right|}$ (the charateristic time appearing in Proposition 3.1) which happens to be equal to $K=\int_{0}^{T^{*}}\left|u_{\infty}\right|$ (the $L^{1}$ norm of $u_{\infty}$ on $\left.\left[0, T^{*}\right]\right)$. We also introduce the $2 \times 2$ matrix $M^{\dagger}=\left(\begin{array}{cc}\left\langle\phi_{1}, B \phi_{1}\right\rangle & \left\langle\phi_{1}, B \phi_{2}\right\rangle \frac{i}{2} \\ \left\langle\phi_{2}, B \phi_{1}\right\rangle \frac{i}{2} & \left\langle\phi_{2}, B \phi_{2}\right\rangle\end{array}\right)$ and the function $v^{*}$ as the reciprocal bijection of $t \mapsto t+\frac{1-\cos \left(\left(\lambda_{2}-\lambda_{1}\right) t\right)}{\lambda_{2}-\lambda_{1}}$.

To begin with, we recall a finite dimensional convergence estimate that will be instrumental in the following:

Proposition 3.2. For any $N$ in $\mathbf{N}$, there exists $K_{N}>$ 0 such that, for every $n$ in $\mathbf{N}$,

$$
\begin{aligned}
& \left\|\pi_{2}^{\Phi} X_{t, s}^{\frac{u_{\infty}}{n},(N)} \pi_{2}^{\Phi}-\exp \left(\left(v^{*}(t)-v^{*}(s)\right) M^{\dagger}\right)\right\| \\
& \leq \frac{K_{N}}{n}\left[v^{*}\left(\frac{t}{n}\right)-v^{*}\left(\frac{s}{n}\right)\right] .
\end{aligned}
$$

Proposition 3.2 follows from a straightforward computation. A proof and the explicit expression of $K_{N}$ can be found in [9, Eq. (12)].

We now come back to the proof of Proposition 3.1. Let $\varepsilon>0$. There exists $N$ in $\mathbf{N}$ such that $\| \pi_{2}^{\Phi} B(1-$ $\left.\pi_{N}\right) \|<\frac{\varepsilon}{K}$. By assumption, there exists $n_{0}$ in $\mathbf{N}$ such that for $n \geq n_{0}$ we have that $\left\|u_{n}-u_{\infty}\right\|_{L^{1}([0, T])}<$ $\left.\varepsilon /(K+1) /\left\|B_{N}^{\Phi}\right\|\right)$. Finally, from (3.9), there exists $n_{1}$ in $\mathbf{N}$ such that, for every $n \geq n_{1}$, for every $s, t$ in $\left[0, n T^{*}\right]$, $\left\|\pi_{2}^{\Phi} X_{t, s}^{\frac{u_{\infty}}{n},(N)} \pi_{2}^{\Phi}-\exp \left(\left(v^{*}(t)-v^{*}(s)\right) M^{\dagger}\right)\right\| \leq \frac{\varepsilon}{K} \quad$ and
$\left\|\left[X_{(t, s)}^{\frac{u_{\infty}}{n},(N)}, \pi_{2}^{\Phi}\right]\right\|<\varepsilon / K$.

Let $n>\max \left(n_{0}, n_{1}, \frac{2 \sqrt{2} K_{N} T^{*}}{\varepsilon}\right)$. There exists a finite sequence $0=t_{0}<t_{1}<\ldots<t_{p}=n T^{*}$ such that $u_{n}$ is constant on each interval $\left(t_{i}, t_{i+1}\right), i=0, \ldots, p-1$. Then

$$
\begin{aligned}
& \left\|\pi_{2}^{\Phi} \Upsilon_{n T^{*}, 0}^{\frac{u_{n}}{n}} \pi_{2}^{\Phi}-\exp \left(K M^{\dagger}\right) \pi_{2}^{\Phi}\right\| \leq \\
& \quad\left\|\pi_{2}^{\Phi}\left(\Upsilon_{n T^{*}, 0}^{\frac{u_{n}}{n}}-X_{n T^{*}, 0}^{\frac{u_{n}}{n},(N)}\right) \pi_{2}^{\Phi}\right\| \\
& \quad+\left\|\pi_{2}^{\Phi}\left(X_{n T^{*}, 0}^{\frac{u_{n}}{n},(N)}-X_{n T^{*}, 0}^{\frac{u_{\infty}}{n},(N)}\right) \pi_{2}^{\Phi}\right\| \\
& \quad+\left\|\pi_{2}^{\Phi} X_{n T^{*}, 0}^{\frac{u_{\infty}}{n},(N)} \pi_{2}^{\Phi}-\exp \left(K M^{\dagger}\right) \pi_{2}^{\Phi}\right\|
\end{aligned}
$$

We give below an estimate of each of the three terms appearing in the RHS of (3.10).

The first term is the most difficult to handle. We first notice that, on each interval $\left(t_{i}, t_{i+1}\right), i<p$, the solution of (2.3) with $u=u_{n} / n$ such that $\psi\left(t_{i}\right) \in$ $\operatorname{span}\left(\phi_{1}^{0}, \phi_{2}^{0}\right)$ is such that, for any $t$ in $\left(t_{i}, t_{i+1}\right)$,

$$
\begin{aligned}
& \pi_{2}^{\Phi} \psi(t)= \\
& \quad \pi_{2}^{\Phi} X_{t, t_{i}}^{\frac{u_{n}}{n},(N)} \psi\left(t_{i}\right) \\
& +\int_{t_{i}}^{t_{i+1}} \frac{u_{n}(s)}{n}\left[\pi_{2}^{\Phi}, X_{t, s}^{\frac{u_{n}}{n},(N)}\right] B\left(1-\pi_{N}\right) \psi(s) \mathrm{d} s \\
& (3.11)+\int_{t_{i}}^{t_{i+1}} \frac{u_{n}(s)}{n} X_{t, s}^{\frac{u_{n}}{n},(N)} \pi_{2}^{\Phi} B\left(1-\pi_{N}\right) \psi(s) \mathrm{d} s
\end{aligned}
$$

The proof of (3.11) follows exactly the computation (3.6) with the difference that since $u_{n}$ is constant on $\left(t_{i}, t_{i+1}\right)$ and $\operatorname{span}\left(\phi_{1}^{0}, \phi_{2}^{0}\right) \subset D\left(A+\frac{u_{n}}{n} B\right), \psi$ is a strong solution of (2.3) and the Duhamel formula is now valid.

A straightforward computation gives, for every $s, t \leq n T^{*}$ :

$$
\begin{aligned}
& \left\|\left[\pi_{2}^{\Phi}, X_{r, s}^{\frac{u_{n}}{n},(N)}\right]\right\| \\
& \quad \leq\left\|\left[\pi_{2}^{\Phi}, X_{r, s}^{\frac{u_{\infty}}{n},(N)}\right]\right\|+2\left\|X_{r, s}^{\frac{u_{\infty}}{n},(N)}-X_{r, s}^{\frac{u_{n}}{n},(N)}\right\| \\
& \quad \leq \frac{\varepsilon}{K}+\left\|B_{N}^{\Phi}\right\| \frac{1}{n} \int_{r}^{s}\left|u_{\infty}-u_{n}\right|(t) \mathrm{d} t
\end{aligned}
$$$$
(3.12) \leq 2 \frac{\varepsilon}{K} \text {. }
$$

As a consequence of (3.11) and (3.12), for every $t$ in $\left(t_{i}, t_{i+1}\right)$,

$$
\begin{aligned}
&\left\|\pi_{2}^{\Phi} \Upsilon_{t, t_{i}}^{\frac{u_{n}}{n}} \pi_{2}^{\Phi}-\pi_{2}^{\Phi} X_{t, t_{i}}^{\frac{u_{n}}{n},(N)} \pi_{2}^{\Phi}\right\| \leq\left(\int_{t_{i}}^{t_{i+1}}\left|\frac{u_{n}(s)}{n}\right| \mathrm{d} s\right) \\
& \times\left[\sup _{r, s \in\left(t_{i}, t_{i+1}\right)}\left\|\left[\pi_{2}^{\Phi}, X_{r, s}^{\frac{u_{n}}{n},(N)}\right]\right\|+\left\|\pi_{2}^{\Phi} B\left(1-\pi_{N}\right)\right\|\right] \\
& \leq \frac{3 \varepsilon}{K} \int_{t_{i}}^{t_{i+1}}\left|u_{n}(s)\right| \mathrm{d} s .
\end{aligned}
$$


One has

$$
\begin{aligned}
\pi_{2}^{\Phi} X_{t, t_{i}}^{\frac{u_{n}}{n},(N)} \pi_{2}^{\Phi}= \\
\left.\quad \pi_{2}^{\Phi} \exp \left(v^{*}\left(\frac{t_{i+1}}{n}\right)-v^{*}\left(\frac{t_{i}}{n}\right)\right) M^{\dagger}\right) \\
\left.\quad+\pi_{2}^{\Phi}\left(X_{t, t_{i}}^{\frac{u_{\infty}}{n}},(N)-\exp \left(v^{*}\left(\frac{t_{i+1}}{n}\right)-v^{*}\left(\frac{t_{i}}{n}\right)\right) M^{\dagger}\right)\right) \pi_{2}^{\Phi} \\
\quad+\pi_{2}^{\Phi}\left(X_{t, t_{i}}^{\frac{u_{n}}{n},(N)}-X_{t, t_{i}}^{\frac{u_{\infty}}{n},(N)}\right) \pi_{2}^{\Phi} .
\end{aligned}
$$

hence

$$
\begin{aligned}
& \left\|\pi_{2}^{\Phi} X_{t, t_{i}}^{\frac{u_{n}}{n},(N)} \pi_{2}^{\Phi}\right\| \geq \\
& \quad 1-\left(\frac{\left\|B_{N}^{\Phi}\right\|}{n} \int_{t_{i}}^{t_{i+1}}\left|u_{n}-u_{\infty}\right|(s) \mathrm{d} s+\frac{2 K_{N}\left|t_{i+1}-t_{i}\right|}{n^{2}}\right)
\end{aligned}
$$

and, since $\sqrt{1-\left(1-u^{2}\right)} \leq \sqrt{2} u$ for $u$ in $[0,1]$,

$$
\begin{aligned}
& \left\|\left(1-\pi_{2}^{\Phi}\right) X_{t, t_{i}}^{\frac{u_{n}}{n},(N)} \pi_{2}^{\Phi}\right\| \leq \\
& \quad \sqrt{2}\left(\frac{\left\|B_{N}^{\Phi}\right\|}{n} \int_{t_{i}}^{t_{i+1}}\left|u_{n}-u_{\infty}\right|(s) \mathrm{d} s+\frac{2 K_{N}\left|t_{i+1}-t_{i}\right|}{n^{2}}\right) .
\end{aligned}
$$

Since $\left\|\Upsilon_{t, t_{i}}^{\frac{u_{n}}{n}} \pi_{2}^{\Phi}\right\|=1$, using Pythagoras Lemma and the property $1-(1-u)^{4} \leq 4 u$ for $u$ in $[0,1]$, one gets

$$
\begin{gathered}
\left\|\left(1-\pi_{2}^{\Phi}\right) \Upsilon_{t i+1, t_{i}}^{\frac{u_{n}}{n}} \pi_{2}^{\Phi}\right\|=\sqrt{1-\left\|\pi_{2}^{\Phi} \Upsilon_{t_{i+1}, t_{i}}^{\frac{u_{n}}{n}} \pi_{2}^{\Phi}\right\|^{2}} \\
\leq \sqrt{1-\left(\left\|\pi_{2}^{\Phi} X_{t, t_{i}}^{\frac{u_{n}}{n},(N)} \pi_{2}^{\Phi}\right\|-\frac{3 \varepsilon}{n K} \int_{t_{i}}^{t_{i+1}}\left|u_{n}(s)\right| \mathrm{d} s\right)^{2}} \\
\leq 1-\left(\left\|\pi_{2}^{\Phi} X_{t, t_{i}}^{\frac{u_{n}}{n},(N)} \pi_{2}^{\Phi}\right\|-\frac{3 \varepsilon}{n K} \int_{t_{i}}^{t_{i+1}}\left|u_{n}(s)\right| \mathrm{d} s\right)^{4} \\
\leq \frac{4}{n}\left(\left\|B_{N}^{\Phi}\right\| \int_{t_{i}}^{t_{i+1}}\left|u_{n}-u_{\infty}\right|+2 \frac{K_{N}}{n}\left|t_{i+1}-t_{i}\right|+\right. \\
\left.\quad+\frac{3 \varepsilon}{K} \int_{t_{i}}^{t_{i+1}}\left|u_{n}\right|\right)
\end{gathered}
$$

Noticing that

$$
\begin{aligned}
& \left\|\pi_{2}^{\Phi} \Upsilon_{n T^{*}, 0}^{\frac{u_{n}}{n}} \pi_{2}^{\Phi}-\pi_{2}^{\Phi} X_{n T^{*}, 0}^{\frac{u_{n}}{n},(N)} \pi_{2}^{\Phi}\right\| \leq \\
& \left\|\pi_{2}^{\Phi}\left(\Upsilon_{t_{p}, t_{p-1}}^{\frac{u_{n}}{n}}-X_{t_{p}, t_{p-1}}^{\frac{u_{n}}{n},(N)}\right) \Upsilon_{t_{p-1}, 0}^{\frac{u_{n}}{n}} \pi_{2}^{\Phi}\right\|+ \\
& \left\|\pi_{2}^{\Phi} X_{t_{p}, t_{p-1}}^{\frac{u_{n}}{n},(N)}\left(\Upsilon_{t_{p-1}, 0}^{\frac{u_{n}}{n}}-X_{t_{p-1}, 0}^{\frac{u_{n}}{n},(N)}\right) \pi_{2}^{\Phi}\right\| \\
& \leq\left\|\pi_{2}^{\Phi}\left(\Upsilon_{t_{p}, t_{p-1}}^{\frac{u_{n}}{n}}-X_{t_{p}, t_{p-1}}^{\frac{u_{n}}{n},(N)}\right) \pi_{2}^{\Phi}\right\|+ \\
& \left\|\pi_{2}^{\Phi}\left(\Upsilon_{t_{p}, t_{p-1}}^{\frac{u_{n}}{n}}-X_{t_{p}, t_{p-1}}^{\frac{u_{n}}{n},(N)}\right)\left(1-\pi_{2}^{\Phi}\right)\right\|+ \\
& \leq \quad\left\|\pi_{2}^{\Phi}\left(\Upsilon_{t_{p}, t_{p-1}}^{\frac{u_{n}}{n}}-X_{t_{p}, t_{p-1}}^{\frac{u_{n}}{n},(N)}\right) \pi_{2}^{\Phi}\right\|+ \\
& \left\|\pi_{2}^{\Phi}\left(\Upsilon_{t_{p}, t_{p-1}}^{\frac{u_{n}}{n}}-X_{t_{p}, t_{p-1}}^{\frac{u_{n}}{n},(N)}\right)\left(1-\pi_{2}^{\Phi}\right)\right\|+ \\
& \left\|\pi_{2}^{\Phi} X_{t_{p}, t_{p-1}}^{\frac{u_{n}}{n},(N)}\left(1-\pi_{2}^{\Phi}\right)\left(\Upsilon_{t_{p-1}, 0}^{\frac{u_{n}}{n}}-X_{t_{p-1}, 0}^{\frac{u_{n}}{n},(N)}\right) \pi_{2}^{\Phi}\right\|+ \\
& \left\|\pi_{2}^{\Phi} X_{t_{p}, t_{p-1}}^{\frac{u_{n}}{n},(N)} \pi_{2}^{\Phi}\left(\Upsilon_{t_{p-1}, 0}^{\frac{u_{n}}{n}}-X_{t_{p-1}, 0}^{\frac{u_{n}}{n},(N)}\right) \pi_{2}^{\Phi}\right\| \\
& \leq\left\|\pi_{2}^{\Phi}\right\| \\
& \left(\Upsilon_{t_{p}, t_{p-1}}^{\frac{u_{n}}{n}}-X_{t_{p}, t_{p-1}}^{\frac{u_{n}}{n},(N)}\right) \pi_{2}^{\Phi} \|+ \\
& \left\|\pi_{2}^{\Phi}\left(\Upsilon_{t_{p}, t_{p-1}}^{\frac{u_{n}}{n}}-X_{t_{p}, t_{p-1}}^{\frac{u_{n}}{n},(N)}\right)\left(1-\pi_{2}^{\Phi}\right)\right\|+ \\
& \left\|\pi_{2}^{\Phi} X_{t_{p}, t_{p-1}}^{\frac{u_{n}}{n},(N)}\left(1-\pi_{2}^{\Phi}\right)\right\|+ \\
& \left\|\pi_{2}^{\Phi}\left(\Upsilon_{t_{p-1}, 0}^{\frac{u_{n}}{n}}-X_{t_{p-1}, 0}^{\frac{u_{n}}{n},(N)}\right) \pi_{2}^{\Phi}\right\|, \\
& \leq
\end{aligned}
$$

one gets after an easy induction

$$
\left\|\pi_{2}^{\Phi} \Upsilon_{n T^{*}, 0}^{\frac{u_{n}}{n}} \pi_{2}^{\Phi}-\pi_{2}^{\Phi} X_{n T^{*}, 0}^{\frac{u_{n}}{n},(N)} \pi_{2}^{\Phi}\right\| \leq \varepsilon(32+4 \sqrt{2}) .
$$

The last two terms of (3.10) are easier to treat, since

$\left\|\pi_{2}^{\Phi}\left(X_{n T^{*}, 0}^{\frac{u_{n}}{n},(N)}-X_{n T^{*}, 0}^{\frac{u_{\infty}}{n},(N)}\right) \pi_{2}^{\Phi}\right\| \leq \frac{\varepsilon}{\left\|B_{N}^{\Phi}\right\|}\left\|B_{N}^{\Phi}\right\|=\varepsilon$

and, by definition of $n_{1}$,

$$
\left\|\pi_{2}^{\Phi} \Upsilon_{n T^{*}, 0}^{\frac{u_{\infty}}{n}} \pi_{2}^{\Phi}-\exp \left(K M^{\dagger}\right) \pi_{2}^{\Phi}\right\| \leq \varepsilon .
$$

Finally,

$$
\left\|\pi_{2}^{\Phi} \Upsilon_{n T^{*}, 0}^{\frac{u_{n}}{n}} \pi_{2}^{\Phi}-\exp \left(K M^{\dagger}\right) \pi_{2}^{\Phi}\right\| \leq \varepsilon(34+4 \sqrt{2}),
$$

and this concludes the proof of Proposition 3.1.

\section{Spectrum perturbation \\ 4.1 The min-max principle}

THEOREM 4.1. If $A$ is a self-adjoint operator on $\mathcal{H}$ bounded from below, $A \geq c I$ in the form sense for some $c \in \mathbf{R}$. Define

$$
\lambda_{n}(A)=\sup _{\phi_{1}, \ldots, \phi_{n-1}} \inf _{\substack{\psi \in\left[\phi_{1}, \ldots, \phi_{n-1}\right]^{\perp} \\ \psi \in Q(A),\|\psi\|=1}}(\psi, A \psi) .
$$

Then, for each $n$, either 
(a) there are $n$ eigenvalues (counting multiplicity) below the bottom of the essential spectrum, and $\lambda_{n}(A)$ is the $n$-th eigenvalue counting multiplicity;

or

(b) $\lambda_{n}(A)$ is the bottom of the essential spectrum, i.e., $\lambda_{n}(A)=\inf \left\{\lambda: \sigma_{\mathrm{ess}}(A)\right\}$ and in this case $\lambda_{n}(A)=$ $\ldots=\lambda_{n+k}(A)=\ldots$ an there are at most $n-1$ eigenvalues below $\lambda_{n}(A)$.

Proof. See [22, Theorem XIII.1]. In [22, Theorem XIII.2] the domain $Q(A)$ is considered in the form sense.

In our example, $Q(A)=H_{0}^{1}((0,+\infty))$ et $Q(A+\epsilon B)=$ $H_{0}^{1}((0,+\infty)) \cap Q(\sqrt{x})$. Notice that the second domain is everywhere dense in the first one for the topology of $H^{1}((0,+\infty))$. Moreover, the exponential decay at infinity of the eigenvectors (see Proposition 2.1) ensures that the eigenvectors of $A$ and $A+\epsilon B$ belong to the domains of $A$ and $A+\epsilon B$.

In the sequel $N:=\left\lfloor 2 D_{e} /\left(h \nu_{0}\right)\right\rfloor-1$ is the number of eigenvalues of $A$ and $\mathcal{L}_{N}^{\Phi}$ the linear space spanned by $\left(\phi_{j}^{0}\right)_{j \in \mathbf{N}}$ a Hilbert basis of $H$ made of eigenvectors of $A$.

Proposition 4.1. Let $(A, B)$ satisfy Assumption 1. Then, for every $n \leq N$, the eigenvalue $\lambda_{n}(A+\epsilon B)$ of $A+\epsilon B$ tends to $\lambda_{n}(A)$ as $\epsilon$ tends to zero.

Proof. Since $\epsilon \in(0,+\infty) \rightarrow \lambda_{n}(A+\epsilon B)$ is nondecreasing, for every $\epsilon>0$, we got $\lambda_{n}(A+\epsilon B) \geq \lambda_{n}(A)$.

Consider now the compression $A_{n}^{\Phi}+\epsilon B_{n}^{\Phi}$ and $\lambda_{k, n}^{\Phi}$ its $k$-the eigenvalue. It is larger than $\lambda_{k}(A+\epsilon B)$ (see [22, Theorem XIII.3]) .

Then, since $\left\|\left|B_{n}^{\Phi} \|\right|\right.$ is finite here for $k \leq n$

$$
\lambda_{k, n}^{\Phi} \leq \lambda_{k}(A)+\epsilon\left\|\mid B_{n}^{\Phi}\right\| \|
$$

and thus

$$
\lambda_{k}(A+\epsilon B) \leq \lambda_{k}(A)+\epsilon\left\|B_{n}^{\Phi}\right\| \|
$$

Hence $\lim _{\epsilon \rightarrow 0} \lambda_{k}(A+\epsilon B)=\lambda_{k}(A)$.

We can even impose the convergence of the eigenvectors.

Proposition 4.2. For $j$ an integer smaller than $N$ the family $\left(\phi_{j}^{\epsilon}\right)_{j \in \mathbf{N}, j \leq N}$ of eigenvectors of $A+\epsilon B$ associated to $\left(\lambda_{j}(A+\epsilon B)\right)_{j \in \mathbf{N}, j \leq N}$ can be chosen, if $\epsilon$ is small enough, such that

$$
\phi_{j}^{\epsilon} \rightarrow \phi_{j}^{0}
$$

as $\epsilon \rightarrow 0$.
Proof. Recall that we write $\lambda_{n}^{\epsilon}$ for $\lambda_{n}(A+\epsilon B)$.

Let us build (up to normalization) $\phi_{j}^{\epsilon}$ as $\phi_{j}^{0}+\eta(\epsilon)$ with $\eta(\epsilon)$ orthogonal to $\phi_{j}^{0}$ smooth and exponentially decaying. The equation $(A+\epsilon B) \phi_{j}^{\epsilon}=\lambda_{j}^{\epsilon} \phi_{j}^{\epsilon}$ leads to

$$
\begin{aligned}
\lambda_{j}+\epsilon\left\langle\phi_{j}^{0}, B\left(\phi_{j}^{0}+\eta(\epsilon)\right)\right\rangle & =\lambda_{j}^{\epsilon} \\
A \eta(\epsilon)+\epsilon \pi_{j}^{\perp} B\left(\phi_{j}^{0}+\eta(\epsilon)\right) & =\lambda_{j}^{\epsilon} \eta(\epsilon)
\end{aligned}
$$

where $\pi_{j}^{\perp}$ is the orthogonal projector onto $\left\{\phi_{j}^{0}\right\}^{\perp}$. The second equation solves as

$$
\eta(\epsilon)=-\epsilon\left(A+\epsilon \pi_{j}^{\perp} B-\lambda_{j}^{\epsilon}\right)^{-1} \pi_{j}^{\perp} B \phi_{j}^{0} .
$$

As long as $j \leq N$ the invertibility of the operators follows from the convergence of the corresponding discrete part of the spectrum exactly as in Proposition 4.1. Notice that plugging the above solution into the first equation leads to fixed point problem that can be solved by means of the implicit function theorem using (1.2). This provides an alternative proof to Proposition 4.1.

4.2 Proof of Proposition 1.1 Since $\lambda_{1}(A+\epsilon B)$ tends to $\lambda_{1}(A)$ and $\lambda_{2}(A+\epsilon B)$ tends to $\lambda_{2}(A)$ as $\epsilon$ tends to zero (Proposition 4.1), $(1,2)$ is a weakly non degenerate transition of $(A+\epsilon B, B)$ for $\epsilon$ small enough. Then, by Proposition 3.1, defining $u: t \mapsto \epsilon+$ $\sin \left(\left(\lambda_{1}^{\epsilon}-\lambda_{2}^{\epsilon}\right) t\right)$, the two sequences $\left(\left|\left\langle\phi_{2}^{\epsilon}, \Upsilon_{n T^{*}, 0}^{u / n} \phi_{1}^{\epsilon}\right\rangle\right|\right)_{n \in \mathbf{N}}$ and $\left(\left|\left\langle\phi_{1}^{\epsilon}, \Upsilon_{n T^{*}, 0}^{u / n} \phi_{2}^{\epsilon}\right\rangle\right|\right)_{n \in \mathbf{N}}$ tend to one. Conclusion follows from the convergence of $\phi_{1}^{\epsilon}$ and $\phi_{2}^{\epsilon}$ to $\phi_{1}^{0}$ and $\phi_{2}^{0}$ respectively as $\epsilon$ tends to zero (Proposition 4.2).

\section{Conclusions}

This note presents an example of positive approximate controllability result for a bilinear quantum dynamics when the free Hamiltonian has a continuous part. The main ingredients of the proof were the min-max principle, for the spectrum perturbation, and the averaging techniques, to obtain controllability.

Unlike most of the recent controllability results for conservative quantum dynamics obtained with geometric techniques, the method used for this particular example heavily depends on the fact that the spectrum of the free Hamiltonian is bounded from below. In particular, the method presented in this note cannot be adapted to the control of bilinear Dirac dynamics.

Nonetheless the provided "black box", applied here in a one dimensional framework with dipolar interaction, can be used in a variety of examples that we think to be of some useful physical interest.

\section{References}


[1] W. O. Amrein, A. Boutet De Monvel, And V. GEORGESCU, $C_{0}$-groups, commutator methods and spectral theory of $N$-body Hamiltonians, vol. 135 of Progress in Mathematics, Birkhäuser Verlag, Basel, 1996.

[2] K. Beauchard, Local controllability of a 1-D Schrödinger equation, J. Math. Pures Appl., 84 (2005), pp. 851-956.

[3] K. Beauchard and J.-M. Coron, Controllability of a quantum particle in a moving potential well, J. Funct. Anal., 232 (2006), pp. 328-389.

[4] K. Beauchard and C. Laurent, Local controllability of $1 D$ linear and nonlinear Schrödinger equations with bilinear control, J. Math. Pures Appl., 94 (2010), pp. 520-554.

[5] K. Beauchard and M. Mirrahimi, Practical stabilization of a quantum particle in a one-dimensional infinite square potential well, SIAM J. Control Optim., 48 (2009), pp. 1179-1205.

[6] K. Beauchard And V. Nersesyan, Semi-global weak stabilization of bilinear Schrödinger equations, C. R. Math. Acad. Sci. Paris, 348 (2010), pp. 1073-1078.

[7] U. Boscain, M. Caponigro, T. Chambrion, And M. SigalotTi, A weak spectral condition for the controllability of the bilinear Schrödinger equation with application to the control of a rotating planar molecule, Comm. Math. Phys., 311 (2012), pp. 423-455.

[8] U. Boscain, M. Caponigro, And M. Sigalotti, Multi-input Schrödinger equation: Controllability, tracking, and application to the quantum angular momentum, Journal of Differential Equations, 256 (2014), pp. $3524-3551$.

[9] T. Chambrion, Periodic excitations of bilinear quantum systems, Automatica J. IFAC, 48 (2012), pp. 20402046.

[10] T. Chambrion, P. Mason, M. Sigalotti, And U. Boscain, Controllability of the discrete-spectrum Schrödinger equation driven by an external field, Ann. Inst. H. Poincaré Anal. Non Linéaire, 26 (2009), pp. 329-349.

[11] E. F. DE Lima And J. E. M. Hornos, Matrix elements for the Morse potential under an external field, Journal of Physics B: Atomic, Molecular and Optical Physics, 38 (2005), p. 815.

[12] P. D. Hislop, Exponential decay of two-body eigenfunctions: a review, in Proceedings of the Symposium on Mathematical Physics and Quantum Field Theory (Berkeley, CA, 1999), vol. 4 of Electron. J. Differ. Equ. Conf., Southwest Texas State Univ., San Marcos, TX, 2000, pp. 265-288 (electronic).

[13] H. M. Hulburt And J. O. Hirschfelder, Potential energy functions for diatomic molecules, The Journal of Chemical Physics, 9 (1941), pp. 61-69.

[14] A. Jensen And G. Nenciu, A unified approach to resolvent expansions at thresholds, Rev. Math. Phys., 13 (2001), pp. 717-754.

[15] R. J. Le Roy, N. S. Dattani, J. A. Coxon, A. J. Ross, P. Crozet, and C. Linton, Accurate analytic potentials for $L i_{2}\left(X^{1} \Sigma_{g}^{+}\right)$and $L i_{2}\left(A^{1} \Sigma_{u}^{+}\right)$from 2 to 90 $\AA$, and the radiative lifetime of $L i(2 p)$, The Journal of Chemical Physics, 131 (2009), p. 204309.

[16] M. Mirrahimi, Lyapunov control of a particle in a finite quantum potential well, in Proceedings of the 45th IEEE Conference on Decision and Control, December 2006.

[17] M. Mirrahimi, P. Rouchon, and G. Turinici, Lyapunov control of bilinear Schrödinger equations, Automatica J. IFAC, 41 (2005), pp. 1987-1994.

[18] P. M. Morse, Diatomic Molecules According to the Wave Mechanics. II. Vibrational Levels, Physical Review, 34 (1929), pp. 57-64.

[19] V. Nersesyan, Growth of Sobolev norms and controllability of the Schrödinger equation, Comm. Math. Phys., 290 (2009), pp. 371-387.

[20] - Global approximate controllability for Schrödinger equation in higher Sobolev norms and applications, Ann. Inst. H. Poincaré Anal. Non Linéaire, 27 (2010), pp. 901-915.

[21] M. ReEd AND B. Simon, Methods of modern mathematical physics. vol. 1. Functional analysis, Academic press New York, 1972.

[22] — Methods of modern mathematical physics. IV. Analysis of operators, Academic Press [Harcourt Brace Jovanovich, Publishers], New York-London, 1978.

[23] J. A. SAnders And F. Verhulst, Averaging methods in nonlinear dynamical systems, Applied mathematical sciences, Springer-Verlag, 1985. 